\title{
Impact of the COVID-19 pandemic on head and neck cancer diagnosis: data from a single referral center, South Tyrol, northern Italy
}

\author{
Luca Gazzini ${ }^{1}$ - Enrico Fazio ${ }^{1}$ Virginia Dallari ${ }^{2}$ - Remo Accorona ${ }^{3} \cdot$ Monir Abousiam $^{1}$ - Aurel Nebiaj ${ }^{1}$. \\ Giovanni Giorgetti ${ }^{1}$. Ilaria Girolami ${ }^{4} \cdot$ Fabio Vittadello $^{4} \cdot$ Roberto Magnato $^{5} \cdot$ Martin Patscheider $^{6}$. \\ Guido Mazzoleni $^{4} \cdot$ Luca Calabrese $^{1}$
}

Received: 8 July 2021 / Accepted: 28 October 2021 / Published online: 5 November 2021

(c) The Author(s), under exclusive licence to Springer-Verlag GmbH Germany, part of Springer Nature 2021

\begin{abstract}
Objective The aim of the study was to evaluate the impact of the COVID-19 pandemic on new diagnoses of head and neck cancer (HNC) in South Tyrol, northern Italy in terms of the number of new diagnoses and worsening disease stage due to diagnostic delay.

Methods Patients were divided into two groups: the control group with a first diagnosis of HNC in 10 months before the national lockdown (March 9th, 2020) and the study group with a first diagnosis of HNC in 10 months after lockdown.

Results A total of 124 patients were included in the study. Before the spread of COVID-19, 79 new diagnoses of HNCs were registered, while in the period after the lockdown, 45 new cancers cases were diagnosed and the difference was statistically significant $(p=0.01278)$. Early clinical T-stage results showed 52 cases in the control group and 21 in the study group, again with a significant difference $(p=0.03711)$. Advanced T-stage results showed 27 cases in the control group and 24 in the study group.

Conclusions This study highlights the impact of the COVID-19 pandemic on HNCs, showing a statistically significant difference in the number of diagnoses before and after the lockdown which was related to the spread of the SARS-CoV-2 virus, and with a relevant decrease in early cT-staged HNCs.
\end{abstract}

Keywords Oncology $\cdot$ Head and neck · Cancers · COVID-19 · Staging · Diagnostic delay

Virginia Dallari

virginia.dallari@gmail.com

1 Division of Otorhinolaryngology, "San Maurizio" Hospital, Bolzano, Italy

2 Division of Otorhinolaryngology, Department of Surgery, Dentistry, Gynecology, and Pediatrics, University Hospital of Verona, University of Verona, Piazzale Aristide Stefani, 1, 37126 Verona, Italy

3 Department of Otorhinolaryngology, Head and Neck Surgery, Fondazione IRCCS Ca' Granda Ospedale Maggiore Policlinico, Milan, Italy

4 Division of Pathological Anatomy, "San Maurizio" Hospital, Bolzano, Italy

5 Division of Otorhinolaryngology, "Franz Tappeiner" Hospital, Merano, Italy

6 Division of Otorhinolaryngology, Bressanone Hospital, Bressanone, Italy

\section{Introduction}

The SARS-CoV-2 pandemic has resulted in thousands of deaths worldwide and has necessitated the rapid redeployment and mobilization of substantial health care resources [1]. Italy was one of the first and most affected European countries. Several strategies have been established by the Italian government to contain the pandemic. Initially, on February 20th 2020, local quarantines were imposed in the most affected areas in the north of the country. Subsequently, stricter lockdown measures were extended nationally from March 9th, 2020 [2].

Although Italy experienced a reduction in the number of COVID-19-positive cases in the summer of 2020, from the end of October, the country faced a second pandemic wave. In this unprecedented situation, the main priority of every health center was to contain the spread of the virus. To this end, the government decided to cut non-emergency health care services $[1,3,4]$, risking the health of patients 
with acute diseases (e.g., cardiovascular, trauma) mainly due to the suspension of regional and hospital emergency care systems. Indeed, oncologic patients requiring time-critical access to health care services have also been seriously affected as a consequence of the pandemic. Screening, timing of work-up, and referral to cancer centers are essential for improved prognosis in oncologic patients [5], including those with head and neck cancers (HNCs).

In most HNC cases, early diagnosis represents the boundary between less and more aggressive surgery, or between resectability and non-resectability of the disease, with a consequent increase in morbidity and mortality [6].

In this study, we analyzed the impact of the SARS-CoV-2 pandemic on new diagnoses and worsening of clinical TNM stage at the referral center for HNCs, Division of Otorhinolaryngology, "San Maurizio" Hospital of Bolzano, where all head and neck oncological cases from the autonomous province of South Tyrol, in northern Italy, are centralized.

South Tyrol represents favorable region to conduct such research both because of higher incidence of HNC comparing to southern regions of Italy, and that the center represents only certified center for oncology by decision of the national and local health authorities.

\section{Materials and methods}

All of the clinical documentation and histological reports from the referral center for HNCs of "San Maurizio" Hospital, in the period between May 2019 and January 2021 were reviewed. All of the patients with a first diagnosis of solid neoplasms in the head and neck district were enrolled in the study.

\section{Inclusion criteria}

- Patients with a first diagnosis of a head and neck solid neoplasm in the nose and paranasal sinuses, nasopharynx, oral cavity, oropharynx, larynx, hypopharynx, salivary glands, or neck lymph node metastases from a cancer of unknown primary (CUP);

- Patients who were permanent residents in the autonomous province of South Tyrol, Italy.

\section{Exclusion criteria}

- Patients in follow-up for HNCs;

- Patients with relapsing HNCs;

- Patients with thyroid neoplasms;

- Patients with head and neck localization of hematological neoplasms;

- Patients with head and neck cutaneous neoplasms;
- Patients referred to the Division of Otorhinolaryngology of "San Maurizio" Hospital from other Italian regions or from abroad.

Depending on the timing of the national lockdown in Italy as a consequence of the SARS-CoV-2 pandemic (March 9th, 2020), patients who met the inclusion criteria were divided into two groups: the control group included patients with a first diagnosis of HNC in 10 months before lockdown, and the study group included patients with a first diagnosis of HNC in 10 months after lockdown.

Demographic data and tumor characteristics, including specific head and neck subsite and stage (clinical classification according to the 8th edition of the TNM Classification of Malignant Tumors [7, 8]) were collected.

The analysis aimed to examine:

(1) The total number of pre- and post-lockdown diagnoses of HNCs.

(2) The clinical T-stage of the tumors diagnosed in the preand post-lockdown periods.

Secondarily, we analyzed the number of diagnoses for every head and neck subsite in the two groups.

\section{Statistical analysis}

This was performed using $\mathrm{R}$ version 3.5.2 software ( $\mathrm{R}$ Core Team (2018). R: A language and environment for statistical computing (R Foundation for Statistical Computing, Vienna, Austria). Univariate analysis of the data was conducted using the $t$ test for normally distributed continuous variables, the Mann-Whitney $U$ test for non-normally distributed continuous variables, and the Chi-squared test or Fisher's exact test for categorical variables. Statistical significance was set at $p<0.05$.

\section{Results}

A total of 124 patients who met the inclusion criteria were included in the study. In the period from May 9th, 2019 to March 8th, 2020, 79 new diagnoses of HNCs were registered, while in the subsequent period from March 9th, 2020 to January 9th, 2021, 45 new cancer cases were diagnosed.

The average rate of new diagnoses in the period before the spread of SARS-CoV-2 was 7 per month, while in the following period, this decreased to 5 per month, and the difference between the groups was statistically significant (Mann-Whitney $U$ test $p=0.01278$ ) (Fig. 1). The trend of new diagnoses in relation to the legal restrictions imposed to limit the spread of the pandemic is shown in Fig. 2. The average age at diagnosis was comparable in the two groups: 


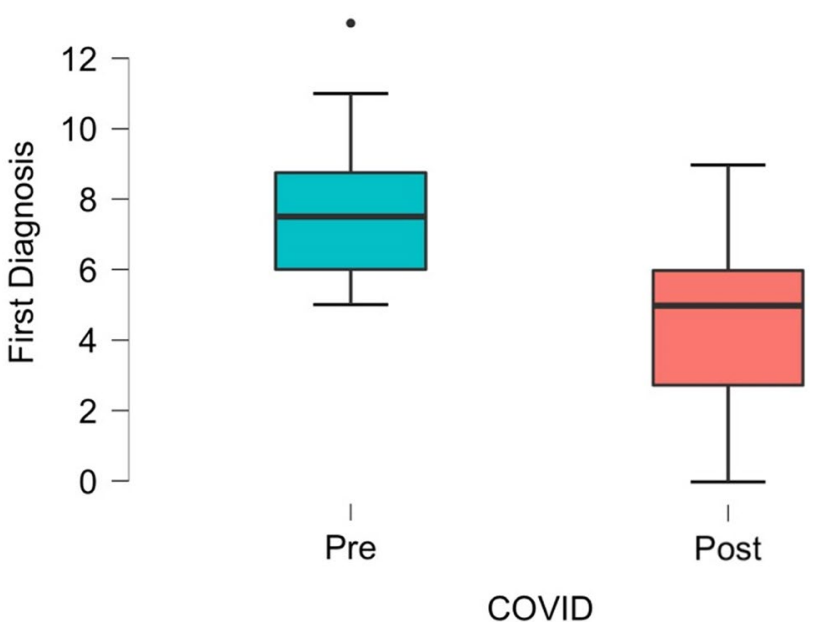

Fig. 1 Incidence of new diagnoses of head and neck cancers per month

70.3 years in the pre-COVID-19 group and 69.4 years in the post-COVID-19 group ( $p=0.70394)$.

The patients were also divided into two groups based on age with 70 years as the cutoff. In the pre-COVID-19 period, 33 patients were younger than 70 and 46 were older than 70, while in the post-COVID-19 period, there were 23 patients younger than 70 and 22 older than 70 . The decrease in the number of diagnoses of HNCs in the post-COVID-19 group older than 70 years was not statistically significantly different from the number of diagnoses in the equivalent pre-COVID-19 group ( $p=0.314992)$. Furthermore, analyzing the age at diagnosis compared with the clinical T-stage (cT), in the control group, there were 25 early cancers and 8 advanced cancers in patients younger than 70 years, and 27 early cancers and 19 advanced cancers in those over 70 years $(p=0.114827)$. In the same analysis performed in the study group, there were 10 early cancers and 13 advanced cancers in the younger patient group and 11 early and 11 advanced cancers in the older patient group ( $p=0.661126)$.

A multivariate analysis using a binary logistic regression model was carried out to evaluate the association between the diagnosis of advanced cancer (cT $\geq 3$ ), the time of diagnosis (pre- and post-COVID-19) and the age of the patient at the time of diagnosis. The time of diagnosis was significantly linked to the diagnosis of advanced cancer $(\mathrm{OR}=2.254,95 \%$ CI $1.06-4.79, p=0.282)$, while the patient's age was not significantly associated $(\mathrm{OR}=1.018$, 95\% CI $0.99-1.05, p=0.282$ ).

Data regarding tumor classification and clinical staging are summarized in Tables 1, 2 and 3, respectively. For patients with an early clinical T-stage (cT1-cT2) and cT0 (CUP metastases), there were 52 cases in the control group and 21 in the study group and the difference between these groups was statistically significant (Chi-squared test $p=0.03711$ ) (Fig. 3). Regarding CUP metastases there were

Table 1 Clinical tumor classification (cT)

\begin{tabular}{lcc}
\hline & Pre-COVID-19 & Post-COVID-19 \\
\hline cT0 & $6(6.9 \%)$ & $0(0 \%)$ \\
cT1 & $21(26.6 \%)$ & $12(26.6 \%)$ \\
cT2 & $25(31.6 \%)$ & $9(20 \%)$ \\
cT3 & $9(11.4 \%)$ & $9(20 \%)$ \\
cT4 & $18(23.8 \%)$ & $15(33.3 \%)$ \\
\hline
\end{tabular}

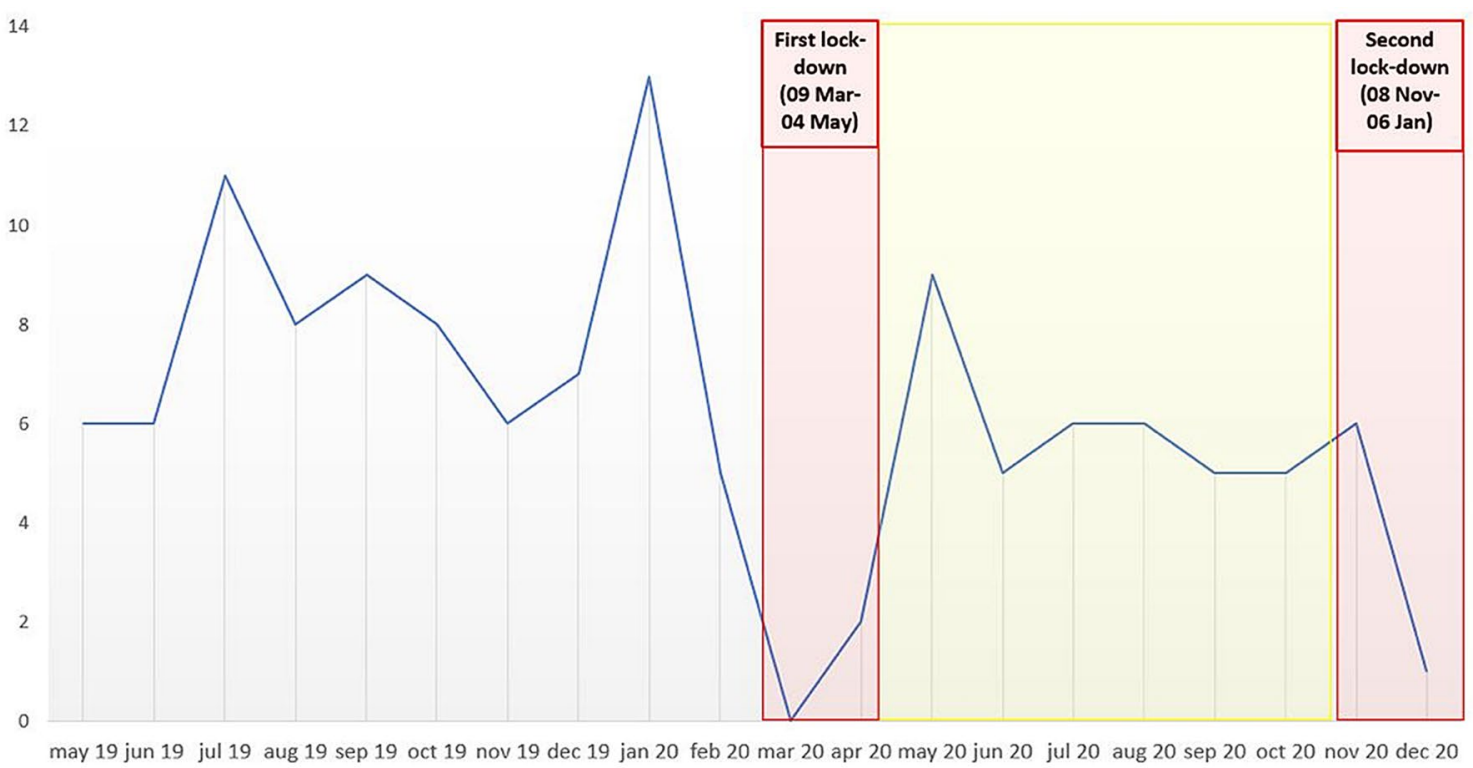

Fig. 2 New diagnoses of head and neck cancers and legal restrictions imposed in Italy to limit the spread of the COVID-19 pandemic 
Table 2 Clinical nodal classification $(\mathrm{cN})$

\begin{tabular}{lcc}
\hline & Pre-COVID-19 & Post-COVID-19 \\
\hline $\mathrm{cN} 0$ & $43(54.4 \%)$ & $29(64.4 \%)$ \\
$\mathrm{cN} 1$ & $8(10.1 \%)$ & $4(8.8 \%)$ \\
$\mathrm{cN} 2$ & $24(30.3 \%)$ & $10(22.2 \%)$ \\
$\mathrm{cN} 3$ & $4(5.0 \%)$ & $2(4.4 \%)$ \\
\hline
\end{tabular}

Table 3 Clinical stage

\begin{tabular}{lcc}
\hline Clinical stage & Pre-COVID-19 & Post-COVID-19 \\
\hline I & $18(24.0 \%)$ & $11(24.4 \%)$ \\
II & $14(17.7 \%)$ & $7(15.5 \%)$ \\
III & $9(11.4 \%)$ & $6(13.3 \%)$ \\
IVA & $25(31.6 \%)$ & $13(28.8 \%)$ \\
IVB & $9(11.4 \%)$ & $5(11.1 \%)$ \\
IVC & $4(5.0 \%)$ & $3(6.6 \%)$ \\
\hline
\end{tabular}

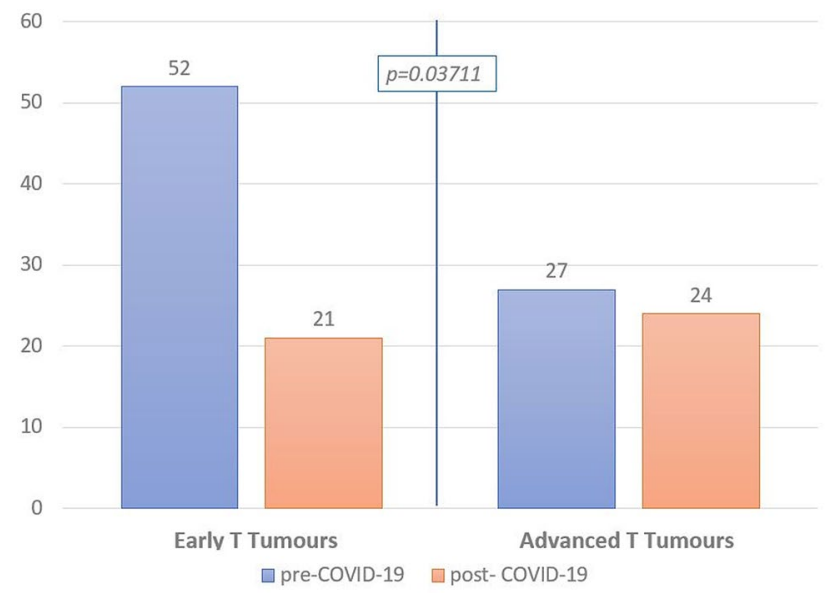

Fig. 3 Incidence of early and advanced head and neck tumors in the control and study groups

six cases in the pre-COVID-19 group (one HPV/p16 related and five non-viral related CUP metastases), whereas no CUP cases were recorded in the post-COVID-19 group.

On the other hand, the number of patients with an advanced T-stage (cT3-cT4) remained stable (27 cases in the control group and 24 in the study group) with no significant difference between the two groups.

Comparing early stage tumors (Stage I and II) in the control group with early stage tumors in the study group, and advanced stage tumors (Stage III and IV) in the control group with advanced stage tumors in the study group, the difference was not statistically significant (Chi-squared test $p=0.6968$ ).

The incidence of new diagnoses split into anatomic subsites is summarized in Fig. 4. Although there were differences in incidence in subsites pre- and post-COVID-19, none were statistically significant. The average time to diagnosis (interval between the first signs and diagnosis) was 64 (15-180) days in the pre-COVID-19 group and 73.5 (7-180) days in the post-COVID-19 group, the difference was not statistically significant (Mann-Whitney $U$ test $p=0.18024$ ). The average time to treatment (interval between diagnosis and treatment) was 28.9 (10-43) days in the pre-COVID-19 group and 30.4 (18-63) days in the post-COVID-19 group, the difference was not statistically significant (Mann-Whitney $U$ test $p=0.77182$ ).

\section{Discussion}

The SARS-CoV-2 infection started in the Chinese region of Wuhan in late 2019, and has had a massive impact in Europe and North America. Daily life changed significantly through the imposition of quarantine and lockdown, and the pandemic had a huge impact on the health systems in many countries. To face this, health systems worldwide had to be rapidly transformed to deal with the unprecedented situation. Health workers were at the center of this transformation, and a large portion were asked to treat COVID-19 patients. This had a knock-on effect on other categories of patients, resulting in an immediate increase in mortality for emergency cases (e.g., cardiovascular diseases, traumas), and a severe diagnostic delay for oncologic patients $[1,3-6,9,10]$. In effect, the number of outpatient visits and instrumental examinations decreased during the pandemic period due to the need for social distancing and the delay in non-urgent investigations [11].

During the Italian lockdown, essential diagnostic services such as oncologic screening were substantially reduced not only because of the reassignment of health care personnel but also because of patients' fear of the hospital environment $[4,5]$. HNC patients experienced a similar fate [11-15]. In a study on 154 Italian Otorhinolaryngology Units, Mannelli et al. noted a marked reduction of more than $80 \%$ in outpatient and surgical services during the COVID-19 crisis in Italy [16].

Surgery is the main course of treatment for most HNCs, and after the outbreak, waiting lists were inevitably prolonged as a result of reallocation of anesthesiologists and reassignment of intensive care units (ICUs) into COVID-19 wards for treatment of the most severe COVID 19 cases. As a result, the delay in diagnosis and treatment could lead to more aggressive surgical procedures, with increasing morbidity and increased risk of recurrence, or to a tumor shift to a non-resectable disease, with an associated increase in mortality [6].

The main purpose of our study was to analyze the impact of the SARS-CoV-2 pandemic on new diagnoses and 


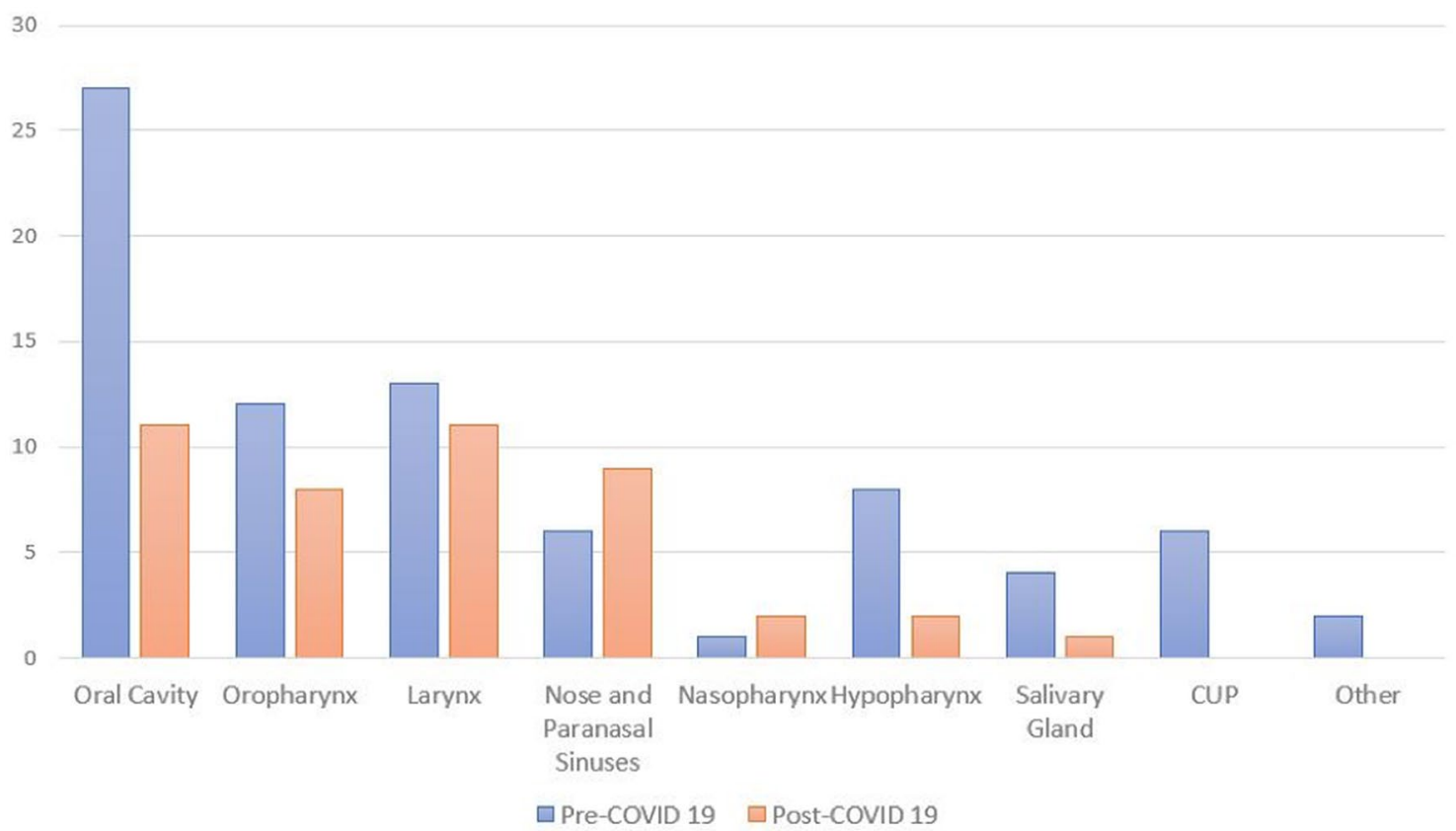

Fig. 4 New diagnoses of head and neck cancers in relation to anatomic subsites

worsening of clinical T-stage of HNCs in a specific area of northern Italy, the autonomous province of South Tyrol.

HNCs represent about $3 \%$ of all malignant tumors in Italy. Approximately 172,000 new cases are diagnosed each year, and most are observed in the male population (approximately 131,000 cases). The annual incidence is $18 / 100,000$ inhabitants, similar to that found in the European Union. The disease is three times more common in the north of the country (34.7/100,000 population) [17].

We believe that South Tyrol is a favorable region to conduct the present research. In fact, "San Maurizio" Hospital of Bolzano is the only certified center for oncology by decision of the national and local health authority, and all cases are referred to this center. Therefore, all of the data in the study period were available for analysis.

To avoid bias due to the drastic change in patient mobility following the restrictions introduced to limit the spread of the pandemic, all HNC patients moving to our referral center from other Italian regions or different countries were excluded from this study. Thyroid cancers, head and neck hematologic neoplasms, and head and neck skin cancers were excluded from the study as all these diseases could potentially have been diagnosed and treated by different specialists. The data of greatest interest in our study were both the number of cases reported to our institute in a matching time frame pre- and post-lockdown, and the $\mathrm{T}$ clinical staging, divided into groups: early $\mathrm{T}$-stage (cT0 and cT1-cT2) and advanced T-stage (cT3-cT4). From our study, we strongly believe that T-stage is the most important parameter in the context of HNCs. Indeed, this is the most important factor prompting patients to seek an assessment at emergency rooms. Our study also included cT0 (CUP metastases) in the early T-stage group as patients with isolated neck swelling often attend surgery after a visit to the GP and/or assessment by ultrasonography, both first-line services heavily undermined by the health crisis and the reason why this study also included cT0 (CUP metastases) in the early T-stage group.

Elderly patients were classified as a high-risk group and were discouraged from attending routine specialist visits during the COVID-19 pandemic. A greater reduction in the number of new diagnoses for these patients could be hypothesized compared with younger patients. Our data showed a greater decline in the number of new diagnoses in the study group in patients older than 70 years (46 patients in the control group and 22 in the study group) compared with the number of new diagnoses in the younger patients which remained stable (23 in the control group and 22 in the study group), but this difference was not statistically significant ( $p=0.314992)$, due to the limited sample number.

Moreover, a multivariate analysis using a binary logistic regression model was carried out to evaluate the association between the diagnosis of advanced cancer (cT $\geq 3)$, the time of diagnosis (pre- and post-COVID-19) and the age of the patient at the time of diagnosis. The time of diagnosis was significantly linked to the diagnosis of advanced cancer $(\mathrm{OR}=2.254,95 \%$ CI $1.06-4.79, p=0.282)$, while the patient's age was not significantly associated $(\mathrm{OR}=1.018$, 95\% CI $0.99-1.05, p=0.282$ ). 
According to our results, we clearly demonstrate a significant impact on the diagnosis of HNCs at our referral center during the COVID-19 pandemic. A statistically significant difference was reported in the total number of new diagnoses of HNCs between the study group and the control group. Furthermore, the trend of diagnoses strongly correlates with tightening of the legal restrictions introduced to limit the spread of the pandemic. During the first strict lockdown (March-April 2020), there was a drastic decrease in the number of new HNC cases reported, with a slight increase in the months immediately after, when the restrictions were eased (Fig. 2). This has occurred despite the fact that urgent visits proceeded as normal and were unaffected during the whole study period, whereas non-urgent visits were cancelled or delayed. From our perspective, the decrease in diagnoses can, therefore, be explained by a lower propensity of patients undergoing a specialist visit because of the pandemic restrictions, and a lower incidence of accidental findings during routine visits. This data is consistent with that reported in the literature. Indeed, several authors have highlighted an evident decrease in the number of hospital visits for myocardial infarction and stroke, related to the peak periods of COVID-19 pandemic spread [1, 3, 9, 10].

Regarding oncologic patients, Mahl and colleagues reported a significant delay in cancer care in up to $58.1 \%$ of cases, due to restrictions in health services during the COVID-19 outbreak in Brazil [18]. Moreover, Dinmohamed and colleagues, analyzing data from the nationwide Netherlands Cancer Registry between February 24 and April 12, 2020 , found a significant decrease in cancer diagnoses when compared with the period before the COVID-19 outbreak and this was observed at almost all cancer sites [19]. Similar results were found by London and colleagues in the USA. Depending on the anatomic subsite, they noted a decrease of between 39.1 and $51.8 \%$ of cancer diagnoses in April 2020 compared to the same period in the previous year [20]. These results are consistent with those reported by Ferrara et al. who compared the number of first pathologic diagnoses of malignancy in weeks 11-20 of 2018, 2019, and 2020 at seven anatomic pathology units serving secondary care hospitals in northern-central Italy, with an overall reduction in diagnosis of $44.9 \%$; however, high-grade tumors were only moderately affected $(-21.7 \%)$ [21].

Interestingly, our data also showed the head and neck subsites which were most affected by the reduction in the number of new diagnoses (such as CUP, salivary gland, oral cavity, and hypopharyngeal cancers) (Fig. 4). The hypopharynx is one of the least symptomatic sites in the head and neck, and patients frequently present with advanced stage cancer. Regarding CUP and salivary gland cancers, as stated before, patients often present with only a swelling of the neck or parotid region. Ultrasound examination is the critical diagnostic tool here, and general practitioners usually convey this to their patients with long-standing swelling, a further basic Italian health service compromised by the pandemic. Finally, patients with early stage cancers of the oral cavity frequently misinterpret tumors as aphthous lesions turning first to a dental service. In our opinion, this underestimation was the reason for the reported diagnostic decrease in early stage cancers in older patients with 27 new diagnoses in the control group and 11 in the study group. On the other hand, we noted a non-significant reduction in the number of new diagnoses of nasal, paranasal sinus, nasopharyngeal cancers with primary symptoms, such as repeated epistaxis, nasal obstruction, cranial nerve impairment and chronic effusive otitis, and also laryngeal cancers with primary symptoms of dysphonia with or without dyspnea. These symptoms would promptly induce these individuals to refer to an emergency department.

Interestingly, this study showed a significant reduction in the diagnosis of early T-stage tumors (cT0+cT1-cT2) in the study group compared to the control group. Moreover, the number of advanced T-stage cancers (cT3-cT4) remained stable (27 in the control group and 24 in the study group). Our findings are in agreement with a preliminary analysis by Mannelli et al. who observed a drastic proportional increase in diagnoses of advanced T-stage HNCs between April and May 2020, when compared with the same period in 2019 (63.5\% and $21.8 \%$, respectively) [22]. We believe that clinical N-stage is less relevant in encouraging patients to refer for treatment, apart from those individuals with very large adenopathies. This can explain the non-significant difference in terms of $\mathrm{cN}$ stage between the pre- and post-lockdown cases, and, ultimately, in terms of the general clinical stage.

The significant reduction in new diagnoses of HNCs related to the COVID-19 outbreak in South Tyrol and demonstrated in this study could also involve other larger health care regions, although the literature-specific data are still preliminary. What we should probably expect in the future is an increase in more advanced cases as a result of the diagnostic delay and an underestimation of the early HNCs. This will likely result in the need for aggressive surgical procedures, with more extensive use of neoadjuvant and adjuvant treatments, increasing the human, and collaterally, the economic costs. Finally, some patients may show progression towards non-resectable disease, and as a consequence, there will be a worsening of prognosis.

To improve the identification of early cancer cases that could have been misdiagnosed during the spread of COVID19 in Italy, there needs to be more collaboration between central hospital departments and local health care professionals. Indeed, it could be useful to equip local ENT specialists and general practitioners with telematic tools so they could introduce and discuss patients having clinical suspicions of HNC with specialists in referral hospitals, even during the COVID-19 pandemic, to reduce delays in diagnosis 
and treatment. Consequently, multidisciplinary meetings involving general practitioners should also be held. Furthermore, it may be useful to introduce online platforms in clinical practice, specifically for medical use, in which general practitioners and local ENT specialists could upload clinical, radiological and endoscopic images. Sharing these data with specialists in referral centers would allow immediate feedback regarding patient status. This practice might allow early consideration of cases by multidisciplinary oncologic head and neck teams for both diagnosis and treatment, avoiding delays due to the reduction of specialist visits in second- and third-level hospitals.

Temesgen and colleagues described their experience in managing outpatients from different medical specialties at the Mayo Clinic during the COVID-19 pandemic [23]. They reported extensive use of telemedicine platforms to manage patients using virtual meetings with videoconferencing and telephone communication. This innovation has been positively accepted by health care professionals and patients. Those authors hypothesized that the availability of telemedicine would encourage individuals to undergo medical evaluation earlier, thus avoiding delayed diagnosis and treatment [23]. Similar positive results are reported in a study by Fassas et al. on managing head and neck oncologic outpatients using telemedicine tools [24].

Despite these positive experiences, the introduction of these tools and online platforms for telehealth has encountered some obstacles in Italy as reported in a recent work by Omboni et al. They state that the poor interconnection between telemedicine services operating at different levels, the lack of a real multidisciplinary approach, and onerous privacy laws can hinder the implementation of effective telemedicine solutions for patient management [25].

The main limitations of our research are the small sample size and the limited geographic area of the study. Our findings need confirmation on a national scale using multicenter studies.

\section{Conclusions}

The results of our research highlight the impact of the COVID-19 pandemic on HNC management in South Tyrol, with a statistically significant difference in the number of diagnoses before and after lockdown related to the spread of the SARS-CoV-2 virus. We have also seen a worsening of the clinical T-stage at diagnosis, with a significant decrease in the diagnosis of early cT-staged HNCs. We believe that, during the COVID-19 pandemic and with the redeployment of medical staff, many early stage HNCs were missed or misdiagnosed and we might expect an increase in more advanced cases in the coming months. To reduce the misdiagnosis of HNCs during the spread of COVID-19, collaboration between central hospital departments and local health care professionals will have to increase.

\section{Declarations}

Conflict of interest The authors declare that they have no conflict of interest. All of the authors have read and approved the manuscript, and none of them have any financial relationships to disclose.

\section{References}

1. Pessoa-Amorim G, Camm CF, Gajendragadkar P et al (2020) Admission of patients with STEMI since the outbreak of the COVID-19 pandemic: a survey by the European Society of Cardiology. Eur Heart J Qual Care Clin Outcomes 6(3):210-216. https://doi.org/10.1093/ehjqcco/qcaa046

2. De Rosa S, Spaccarotella C, Basso C et al (2020) Reduction of hospitalizations for myocardial infarction in Italy in the COVID19 era. Eur Heart J 41(22):2083-2088. https://doi.org/10.1093/ eurheartj/ehaa409

3. Tejada Meza H, Lambea Gil Á, Sancho Saldaña A et al (2020) Impact of COVID-19 outbreak in reperfusion therapies of acute ischaemic stroke in northwest Spain. Eur J Neurol 27(12):24912498. https://doi.org/10.1111/ene. 14467

4. Maringe C, Spicer J, Morris M et al (2020) The impact of the COVID-19 pandemic on cancer deaths due to delays in diagnosis in England, UK: a national, population-based, modelling study. Lancet Oncol 21(8):1023-1034. https://doi.org/10.1016/S14702045(20)30388-0

5. Jones D, Neal RD, Duffy SRG et al (2020) Impact of the COVID19 pandemic on the symptomatic diagnosis of cancer: the view from primary care. Lancet Oncol 21(6):748-750. https://doi.org/ 10.1016/S1470-2045(20)30242-4

6. Salari A, Jalaeefar A, Shirkhoda M (2020) What is the best treatment option for head and neck cancers in COVID-19 pandemic? A rapid review. Am J Otolaryngol 41(6):102738. https://doi.org/ 10.1016/j.amjoto.2020.102738

7. Brierley J, Gospodarowicz M, Wittekind C (2017) UICC TNM classification of malignant tumours, 8th edn. Wiley, Chichester

8. Amin M, Edge S, Greene F et al (2017) AJCC cancer staging manual, 8th edn. Springer, New York

9. Solomon MD, McNulty EJ, Rana JS et al (2020) The Covid-19 pandemic and the incidence of acute myocardial infarction. $\mathrm{N}$ Engl J Med 383(7):691-693. https://doi.org/10.1056/NEJMc 2015630

10. De Filippo O, D'Ascenzo F, Angelini F et al (2020) Reduced rate of hospital admissions for ACS during Covid-19 outbreak in Northern Italy. N Engl J Med 383(1):88-89. https://doi.org/10. 1056/NEJMc2009166

11. Mehanna H, Hardman JC, Shenson JA et al (2020) Recommendations for head and neck surgical oncology practice in a setting of acute severe resource constraint during the COVID-19 pandemic: an international consensus. Lancet Oncol 21(7):e350-e359. https://doi.org/10.1016/S1470-2045(20)30334-X

12. MD Anderson Head and Neck Surgery Treatment Guidelines Consortium, Consortium members, Maniakas A et al (2020) Head and neck surgical oncology in the time of a pandemic: subsite-specific triage guidelines during the COVID-19 pandemic. Head Neck 42(6):1194-1201. https://doi.org/10.1002/hed.26206

13. Brody RM, Albergotti WG, Shimunov D et al (2020) Changes in head and neck oncologic practice during the COVID-19 
pandemic. Head Neck 42(7):1448-1453. https://doi.org/10.1002/ hed.26233

14. Topf MC, Shenson JA, Holsinger FC et al (2020) Framework for prioritizing head and neck surgery during the COVID-19 pandemic. Head Neck 42(6):1159-1167. https://doi.org/10.1002/hed. 26184

15. Kowalski LP, Sanabria A, Ridge JA et al (2020) COVID-19 pandemic: effects and evidence-based recommendations for otolaryngology and head and neck surgery practice. Head Neck 42(6):1259-1267. https://doi.org/10.1002/hed.26164

16. Mannelli G, Ralli M, Bonali M et al (2020) Impact of COVID-19 pandemic on Italian Otolaryngology Units: a nationwide study. Acta Otorhinolaryngol Ital 40(5):325-331. https://doi.org/10. 14639/0392-100X-N0832

17. AIOM/AIRTUM (2018) I numeri del cancro in Italia. Intermedia Editore, Brescia

18. Mahl C, Melo LRS, Almeida MHA et al (2020) Delay in head and neck cancer care during the COVID-19 pandemic and its impact on health outcomes. Braz Oral Res 34:e126. https://doi.org/10. 1590/1807-3107bor-2020.vol34.0126 (published 2020 Dec 18)

19. Dinmohamed AG, Visser O, Verhoeven RHA et al (2020) Fewer cancer diagnoses during the COVID-19 epidemic in the Netherlands [published correction appears in Lancet Oncol. 2020 May 4]. Lancet Oncol 21(6):750-751. https://doi.org/10.1016/S14702045(20)30265-5

20. London JW, Fazio-Eynullayeva E, Palchuk MB et al (2020) Effects of the COVID-19 pandemic on cancer-related patient encounters.
JCO Clin Cancer Inform 4:657-665. https://doi.org/10.1200/CCI. 20.00068

21. Ferrara G, De Vincentiis L, Ambrosini-Spaltro A et al (2021) Cancer diagnostic delay in Northern and Central Italy during the 2020 lockdown due to the coronavirus disease 2019 pandemic. Am J Clin Pathol 155(1):64-68. https://doi.org/10.1093/ajcp/aqaa177

22. Mannelli G, Santoro R, Bonomo P et al (2020) Will a tumor pandemic come after the COVID-19 pandemic? Head and neck cancer perspective. Eur Rev Med Pharmacol Sci 24(16):8573-8575. https://doi.org/10.26355/eurrev_202008_22653

23. Temesgen ZM, DeSimone DC, Mahmood M et al (2020) Health care after the COVID-19 pandemic and the influence of telemedicine. Mayo Clin Proc 95(9S):S66-S68. https://doi.org/10.1016/j. mayocp.2020.06.052

24. Fassas S, Cummings E, Sykes KJ et al (2021) Telemedicine for head and neck cancer surveillance in the COVID-19 era: promise and pitfalls [published online ahead of print, 2021 Mar 4]. Head Neck. https://doi.org/10.1002/hed.26659

25. Omboni S (2020) Telemedicine during the COVID-19 in Italy: a missed opportunity? Telemed J E Health 26(8):973-975. https:// doi.org/10.1089/tmj.2020.0106

Publisher's Note Springer Nature remains neutral with regard to jurisdictional claims in published maps and institutional affiliations. 